\title{
Prospective evaluation of the proportion of sessile serrated adenoma/polyps in endoscopically diagnosed colorectal polyps with hyperplastic features ${ }^{1}$
}

Authors

Institutions
Wataru Sano ${ }^{1}$, Yasushi Sano ${ }^{1}$, Mineo Iwatate ${ }^{1}$, Noriaki Hasuike ${ }^{1}$, Santa Hattori ${ }^{1}$, Hidekazu Kosaka' ${ }^{1}$ Taro Ikumoto ${ }^{1}$, Masahito Kotaka ${ }^{1}$, Takahiro Fujimori ${ }^{2}$

${ }^{1}$ Gastrointestinal Center, Sano Hospital, Hyogo, Japan

${ }^{2}$ Department of Pathology, Shinko Hospital, Hyogo, Japan submitted

28. November 2014

accepted after revision

\section{Bibliography}

DOI http://dx.doi.org/

10.1055/s-0034-1391948

Published online: 5.5.2015

Endosc Int Open 2015; 03:

E354-E358

(c) Georg Thieme Verlag KG Stuttgart · New York

E-ISSN 2196-9736

\section{Corresponding author}

\section{Wataru Sano, MD}

Gastrointestinal Center

Sano Hospital

2-5-1 Shimizugaoka

Tarumi

Kobe

Hyogo 655-0031

Japan

Fax: +81-78-7850077

watasano@yahoo.co.jp
2. March 2015

Background and study aims: Sessile serrated adenoma/polyps (SSA/Ps) are considered precursors of colorectal cancers with microsatellite instability. However, it is still difficult to differentiate SSA/Ps from hyperplastic polyps endoscopically; therefore, the prevalence of SSA/Ps remains uncertain in clinical practice. This study aimed to clarify the proportion of SSA/Ps in endoscopically diagnosed colorectal polyps with hyperplastic features (E-HPs).

Patients and methods: Patients aged $\geq 40$ years undergoing colonoscopy for standard clinical indications at our center were prospectively enrolled between June 2013 and May 2014. During colonoscopy, $0.05 \%$ indigo carmine dye was sprayed throughout the colorectum to highlight lesions. All detected lesions were diagnosed by high definition magnifying narrow-band imaging and were resected endoscopically or surgically, apart from rectosigmoid E-HPs $\leq 5 \mathrm{~mm}$. The number of

\section{Introduction}

\section{$\nabla$}

In recent years, the serrated neoplastic pathway arising from serrated lesions has attracted much attention as a new colorectal carcinogenic pathway by which approximately $20 \%$ of all colorectal cancers are believed to develop. At present, colorectal serrated lesions are classified as hyperplastic polyps (HPs), sessile serrated adenoma/polyps (SSA/Ps) with or without cytological dysplasia, or traditional serrated adenomas (TSAs) according to the World Health Organization criteria. Of these serrated lesions, SSA/Ps and TSAs are considered premalignant, and SSA/Ps are believed to be the most potent precursors of colorectal cancers with microsatellite instability because of their genetic backgrounds including BRAF gene mutations and hypermethylation of $\mathrm{CPG}$ islands

\section{License terms}

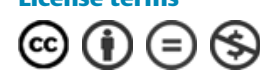

${ }^{1}$ Presented in part as a free paper at the Asian Pacific Digestive Week, 23 November 2014, Bali, Indonesia. rectosigmoid E-HPs $\leq 5 \mathrm{~mm}$ was recorded, and some were resected for use as tissue samples.

Results: A total of 343 patients (male: 42.9\%; mean age: 61.5 years) were included. Among 3838 E-HPs (distal: 96.4\%) detected in 294 patients, 792 were resected and analyzed. All of 21 SSA/Ps identified in 17 patients were included in E-HPs, and the overall proportion of SSA/Ps in EHPs was $2.7 \%$. However, this proportion increased with the size of E-HPs ( $\leq 5 \mathrm{~mm}$ : $0.7 \% ; 6-9 \mathrm{~mm}$ : $29.0 \%$; $\geq 10 \mathrm{~mm}: 70 \%$ ) and was higher in the proximal colon than in the distal colorectum $(10.9 \%$ vs. $0.9 \%$ ). In addition, no SSA/P was found in the rectum, and no SSA/P had cytological dysplasia. Conclusions: The overall proportion of SSA/Ps in E-HPs was $2.7 \%$, although this proportion was higher in the proximal colon and increased with the size of E-HPs. SSA/Ps were common in routine colonoscopy, with a prevalence of at least $5.0 \%$. Study registration: UMIN000010832.

in the promoter regions of tumor suppressor genes (i.e. the $\mathrm{CpG}$ island methylator phenotype). Recent image enhancement techniques have improved the diagnostic accuracy of differential diagnosis between neoplastic and non-neoplastic colorectal lesions by $>90 \%$ in real-time histology $[1,2]$. Of the serrated lesions, TSAs are commonly reddish, pinecone- or branch coral-shaped, protruded lesions with a type $\mathrm{III}_{\mathrm{H}}$ (fern-like) or $\mathrm{IV}_{\mathrm{H}}$ (pinecone-like) pit pattern [3]; thus, it is not difficult to distinguish them from other serrated lesions and diagnose them as neoplasia. Conversely, most SSA/Ps are normal in color or discolored flat lesions similar to HPs; thus, SSA/Ps may often be misdiagnosed as HPs. Although the endoscopic features of SSA/Ps, such as a type II-O pit pattern and a mucous cap, have been reported over recent years $[4,5]$, it remains difficult to differentiate SSA/Ps from HPs even by image-enhanced endoscopy. 
Therefore, we designed this study to clarify the proportion of SSA/Ps in endoscopically diagnosed colorectal polyps with hyperplastic features (E-HPs). We believe that elucidation of the proportion of SSA/Ps in E-HPs will enhance current knowledge with regard to SSA/Ps and advance the treatment of E-HPs.

\section{Patients and methods \\ $\nabla$}

\section{Patients and exclusion criteria}

A total of 364 patients aged $\geq 40$ years undergoing colonoscopy for standard clinical indications at our center were prospectively enrolled in this study between June 2013 and May 2014 († Fig. 1). Patients with a history of polypectomy for colorectal serrated lesions or surgical colorectal resection, patients with inflammatory bowel disease or colorectal polyposis, and patients with poor bowel preparation or incomplete colonoscopy were excluded. In addition, informed consent was obtained from all patients, apart from six patients; thus, they were also excluded.

The study protocol was approved by the local institutional review board (SH09-13). The clinical trial was registered in the University Hospital Medical Information Network Center (UMIN000010832).

\section{Colonoscopy}

All patients received $1.8 \mathrm{~L}$ of isotonic magnesium citrate solution for bowel preparation. Colonoscopies were performed by six experienced gastrointestinal endoscopists using a high definition magnifying video colonoscope equipped with a waterjet function (CF-HQ290I, PCF-Q260AZI, EVIS 290 system; Olympus Medical Systems, Tokyo, Japan). During colonoscopy, 0.05\% indigo carmine solution was sprayed throughout the colorectum to highlight lesions using the waterjet function. All detected lesions were diagnosed by magnifying narrow-band imaging (NBI), according to the NBI international colorectal endoscopic classification $[6,7]$, and were resected endoscopically or surgically, apart from rectosigmoid E-HPs $\leq 5 \mathrm{~mm}$. The number of rectosigmoid E-HPs $\leq 5 \mathrm{~mm}$ was recorded by a tally counter, and some were resected endoscopically for use as tissue samples to confirm the quality assurance of real-time histology by magnifying colonoscopy. Polyps $\leq 2 \mathrm{~mm}$ were removed using cold biopsy forceps in a single bite, and those $\geq 3 \mathrm{~mm}$ were resected by a hot snare procedure. All lesions were measured by comparison with open or closed biopsy forceps or polypectomy snare. Cecal intubation was confirmed by identification of the appendiceal orifice. Withdrawal time was recorded using a stopwatch after subtracting the time needed for biopsy or polypectomy. The proximal colon was defined as proximal to the splenic flexure, i.e. cecum, ascending colon, and transverse colon.

\section{Histopathology}

Resected specimens were evaluated by an experienced gastrointestinal pathologist (T.F.) who was a member of the Japanese Society for Cancer of the Colon and Rectum (JSCCR) and was well versed in the diagnosis of colorectal serrated lesions. Histopathological diagnosis was based on the morphological features on hematoxylin and eosin staining. Of the serrated lesions, HPs were defined by a serrated epithelial architecture in the upper part of the crypts, proliferative zones limited to the lower part of the crypts, and the absence of cytological dysplasia. SSA/Ps were distinguished from HPs by the following JSCCR criteria: (1) crypt dilation, (2) irregularly branching crypts, and (3) horizontally ar-

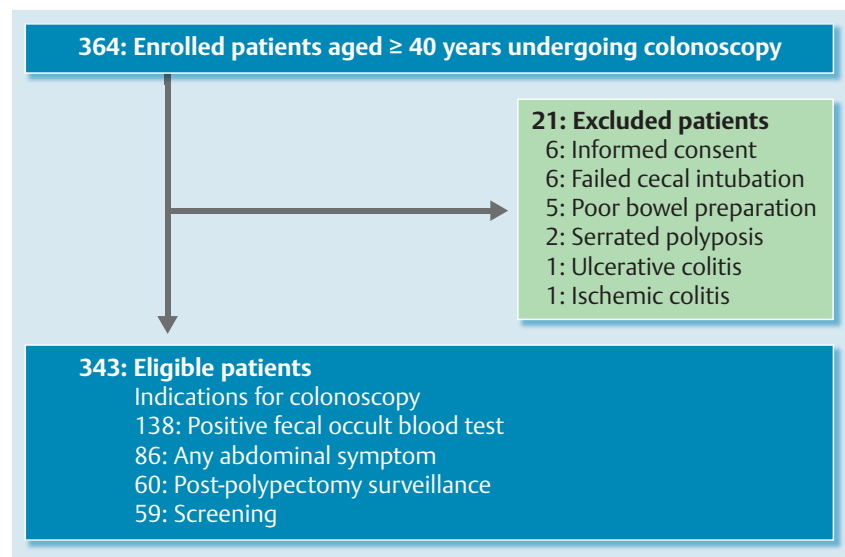

Fig. 1 Patient flow chart.

ranged basal crypts (inverted T- and/or L-shaped crypts) [8,9]. When at least two of these three findings were seen in the areas of $\geq 10 \%$ of the lesions, the serrated lesions were diagnosed as SSA/Ps. However, the serrated lesions which did not meet the above criteria were diagnosed as HPs. TSAs were defined by villiform growth, serrated epithelium in association with nuclear and cytologic atypia, ectopic crypt formation, and eosinophilic cytoplasm.

After the study, all resected specimens of SSA/Ps and TSAs intermingled with some resected specimens of HPs and conventional adenomas were reevaluated by the same pathologist to confirm the final pathological diagnoses. At this time, the pathologist was blinded to the clinical data, such as the endoscopic diagnosis, the initial pathological diagnosis, and the anatomical location, size, and shape of the lesions.

\section{Statistical analysis}

Differences in the proportion of SSA/Ps in E-HPs were assessed using the chi-squared test or Fisher's exact test, as appropriate. Two-sided $P$ values of $<0.05$ were considered statistically significant.

\section{Results \\ $\nabla$}

\section{Patients and colonoscopy}

A total of 343 eligible patients (male: $42.9 \%$ ) with a mean age of 61.5 years (range: $40-86$ years) were included in this study ( 0 Fig. 1). The indications for colonoscopy were positive fecal occult blood test $(n=138)$, any abdominal symptom $(n=86)$, postpolypectomy surveillance $(n=60)$, and screening $(n=59)$. The mean and median withdrawal times during colonoscopy were $9.5 \mathrm{~min}$ and $8.9 \mathrm{~min}$, respectively. The adenoma detection rate was $56.0 \%$.

\section{Histopathology}

A total of 1301 lesions were resected endoscopically or surgically and then analyzed histopathologically. Among them, 21 were SSA/Ps, 13 were TSAs, 472 were conventional adenomas, 29 were adenocarcinomas (intramucosal: 16; invasive: 13 ), 1 was a neuroendocrine tumor (grade 1), and 2 were juvenile polyps. The remaining 763 lesions were HPs, hyperplastic nodules, or colorectal mucosa. 


\begin{tabular}{|lccc|}
\hline Size and histopathology & Proximal colon & Distal colon & Rectum \\
\hline$\leq 5 \mathrm{~mm}, \mathrm{n}$ & 114 & 1954 & 1729 \\
$\mathrm{HP} / \mathrm{HN} / \mathrm{CM}, \mathrm{n}(\%)$ & $78(68.4)$ & $305(94.1)$ & $309(98.7)$ \\
$\mathrm{SSA} / \mathrm{P}, \mathrm{n}(\%)$ & $2(1.8)$ & $3(0.9)$ & $0(0)$ \\
TSA, $\mathrm{n}(\%)$ & $1(0.9)$ & $0(0)$ & $1(0.3)$ \\
Conventional adenoma, $\mathrm{n}(\%)$ & $33(28.9)$ & $16(4.9)$ & $3(1.0)$ \\
\hline Not resected, $\mathrm{n}(\%)$ & & 1630 & 1416 \\
\hline 6-9 mm, & 16 & 9 & 6 \\
\hline HP/HN/CM, $\mathrm{n}(\%)$ & $8(50)$ & $7(77.8)$ & $6(100)$ \\
SSA/P, $\mathrm{n}(\%)$ & $7(43.8)$ & $2(22.2)$ & $0(0)$ \\
TSA, $\mathrm{n}(\%)$ & $1(6.3)$ & $0(0)$ & $0(0)$ \\
Conventional adenoma, $\mathrm{n}(\%)$ & $0(0)$ & $0(0)$ & $0(0)$ \\
\hline$\geq 10 \mathrm{~mm}, \mathrm{n}$ & 7 & 2 & 1 \\
HP/HN/CM, $\mathrm{n}(\%)$ & $1(14.3)$ & $1(50)$ & $1(100)$ \\
SSA/P, $\mathrm{n}(\%)$ & $6(85.7)$ & $1(50)$ & $0(0)$ \\
TSA, $\mathrm{n}(\%)$ & $0(0)$ & $0(0)$ & $0(0)$ \\
Conventional adenoma, $\mathrm{n}(\%)$ & $0(0)$ & $0(0)$ & $0(0)$ \\
\hline Total, $\mathrm{n}$ & 137 & 1965 & 1736 \\
\hline
\end{tabular}

Table 1 Histopathology of endoscopically diagnosed colorectal polyps with hyperplastic features (E-HPs).

Among 3838 E-HPs detected in 294 of 343 patients, 792 were endoscopically resected and histopathologically analyzed. HP, hyperplastic polyp; HN, hyperplastic nodule; CM, colorectal mucosa; SSA/P, sessile serrated adenoma/polyp; TSA, traditional serrated adenoma.

${ }^{1}$ Among 3683 distal E-HPs $\leq 5$ mm, 3046 rectosigmoid E-HPs were not resected.

\section{Prevalence of E-HPs}

A total of 3838 lesions were endoscopically diagnosed as HPs ( $\bullet$ Table 1). The overall prevalence of E-HPs, defined as the proportion of patients with $\geq 1 \mathrm{E}-\mathrm{HP}$, was $85.7 \%$ of 343 patients. The prevalence of E-HPs $\leq 5 \mathrm{~mm}, 6-9 \mathrm{~mm}$, and $\geq 10 \mathrm{~mm}$ was $85.7 \%$, $6.1 \%$, and $2.6 \%$, respectively. The prevalence of proximal and distal E-HPs was $23.9 \%$ and $85.1 \%$, respectively. In addition, $13.6 \%$ and $13.0 \%$ of conventional adenomas $\leq 5 \mathrm{~mm}$ were misdiagnosed as HPs in the proximal and distal colorectum, respectively.

\section{Prevalence of SSA/Ps}

A total of 21 SSA/Ps were identified in 17 of 343 patients, and the prevalence of SSA/Ps, defined as the proportion of patients with $\geq 1 \mathrm{SSA} / \mathrm{P}$, was $5.0 \%$. However, there was no SSA/P with cytological dysplasia in 343 patients (95\% confidence interval (CI): $0-0.9$ ). - Fig. 2 shows an example of an SSA/P identified and resected endoscopically in this study.

\section{Proportion of SSA/Ps in E-HPs}

Among 3838 E-HPs (distal: 96.4\%), 792 were endoscopically resected and histopathologically analyzed. All of 21 SSA/Ps were included in E-HPs, and the overall proportion of SSA/Ps in 792 E-HPs was $2.7 \%$ ( Table 2). However, this proportion significantly increased with the size of E-HPs ( $\leq 5 \mathrm{~mm}$ : $0.7 \%$; $6-9 \mathrm{~mm}$ : $29.0 \%$; $\geq 10 \mathrm{~mm}: 70 \%$ ) and was significantly higher in the proximal colon than in the distal colorectum (10.9\% vs. $0.9 \%$ ). In addition, no SSA/P was found in the rectum.

\section{Diagnostic accuracy}

The diagnostic accuracy of differential diagnosis between neoplasia and non-neoplasia for all resected lesions was 92.2\% [sensitivity: $89.1 \%$; specificity: $94.3 \%$; positive predictive value (PPV): 91.1\%; negative predictive value (NPV): $93.0 \%]$, and that for rectosigmoid lesions resected endoscopically was $95.5 \%$ (sensitivity: 85.4\%; specificity: 97.4\%; PPV: 86.8\%; NPV: 97.1\%).

\section{Discussion \\ $\nabla$}

To the best of our knowledge, this is the first prospective study to examine the proportion of SSA/Ps in E-HPs and the prevalence of SSA/Ps in Japanese patients undergoing routine colonoscopy, using pan-colonic chromoendoscopy with high definition magnifying NBI colonoscopy.

Previous studies have demonstrated that pan-colonic chromoendoscopy with indigo carmine dye improves the detection of colorectal polyps, including serrated lesions, compared with conventional colonoscopy [10-12]. Furthermore, the diagnostic accuracy of magnifying NBI colonoscopy in differential diagnosis between neoplastic and non-neoplastic colorectal lesions is similar to that of magnifying chromoendoscopy but greater than that of conventional colonoscopy [2]. Therefore, in this study, we used pan-colonic chromoendoscopy to detect lesions and magnifying NBI colonoscopy, which is simpler and more convenient than
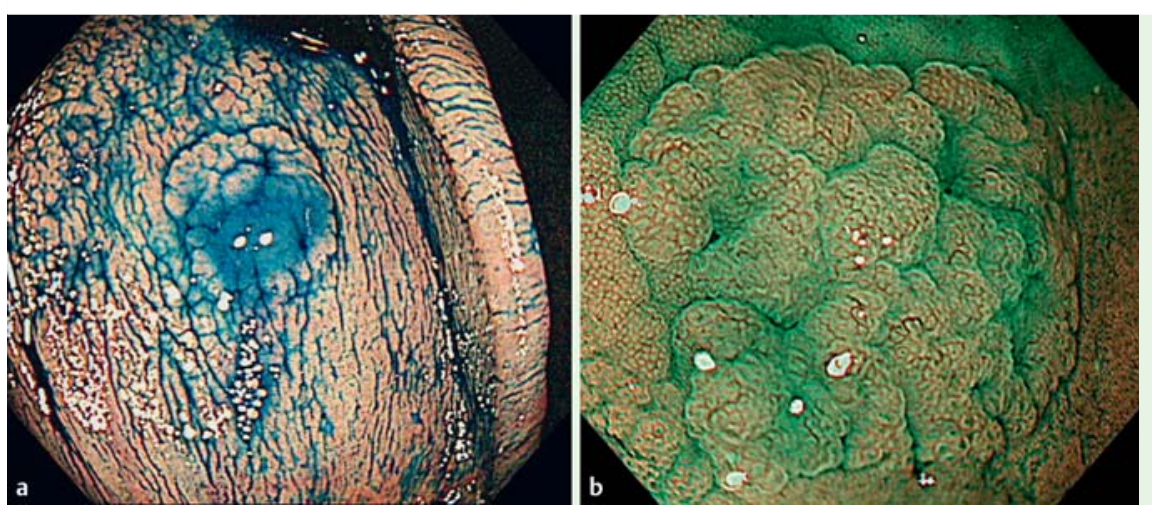

Fig. 2 An example of a sessile serrated adenoma/ polyp. a Non-magnified view of chromoendoscopy with $0.05 \%$ indigo carmine dye. A flat lesion, $7 \mathrm{~mm}$ in diameter, with mucus was seen in the ascending colon. b Magnified view of narrow-band imaging colonoscopy. The lesion showed the same color as the background, slightly thicker brown vessels, and a slightly irregular surface. 


\begin{tabular}{|llll|}
\hline Size & Proximal colon & Distal colorectum & Total \\
\hline$\leq 5 \mathrm{~mm}, \%(95 \% \mathrm{Cl})$ & 1.8 & 0.5 & 0.7 \\
& $(0.2-6.2)$ & $(0.1-1.4)$ & $(0.2-1.5)$ \\
\hline $6-9 \mathrm{~mm}, \%(95 \% \mathrm{Cl})$ & 43.8 & 13.3 & $29.0^{1}$ \\
& $(19.8-70.1)$ & $(1.7-40.5)$ & $(14.2-48.0)$ \\
\hline$\geq 10 \mathrm{~mm}, \%(95 \% \mathrm{Cl})$ & 85.7 & 33.3 & $(34.8-93.3)$ \\
\hline Total, \% $(95 \% \mathrm{Cl})$ & $(42.1-99.6)$ & $(0.8-90.6)$ & 2.7 \\
& $10.9^{3}$ & 0.9 & $(1.7-4.0)$ \\
\hline
\end{tabular}

Table 2 Proportion of sessile serrated adenoma/polyps (SSA/ Ps) in endoscopically diagnosed colorectal polyps with hyperplastic features (E-HPs).

All $21 \mathrm{SSA} / \mathrm{Ps}$ identified in 17 patients were included in $\mathrm{E}-\mathrm{HPs}$, and the overall proportion of SSA/Ps in $792 \mathrm{E}-\mathrm{HPs}$ was $2.7 \%$. There was no SSA/P with cytological dysplasia.

${ }^{1} P<0.01$ vs. $\leq 5 \mathrm{~mm}$

$2 P<0.05$ vs. $6-9 \mathrm{~mm}$

${ }_{3} P<0.01$ vs. distal; chi-squared test or Fisher's exact test.

chromoendoscopy, to arrive at a diagnosis, and then analyzed the resected lesions histopathologically.

In previous studies, indigo carmine solution has been used at concentrations ranging from $0.05 \%$ to $0.4 \%$ for pan-colonic chromoendoscopy [10-13]. Although we routinely use $0.2 \%$ indigo carmine solution to observe colorectal lesions during chromoendoscopy at our center, we found that $0.2 \%$ indigo carmine solution affected the NBI observation in a pretest before the study. Hence, in this study, we used $0.05 \%$ indigo carmine solution, which was sufficient to highlight lesions and did not affect the NBI observation in the pretest.

Yano et al. clarified the prevalence and distribution of varioussized E-HPs by pan-colonic chromoendoscopy with $0.05 \%$ indigo carmine dye [13]. In their prospective study, 3060 E-HPs were detected in 226 of 263 patients, and the prevalence of E-HPs was $85.9 \%$. Moreover, E-HPs $6-9 \mathrm{~mm}$ and those $\geq 10 \mathrm{~mm}$ were predominantly located in the proximal colon compared with E-HPs $\leq 5 \mathrm{~mm}$. These findings are consistent with the results of the present study ( $\bullet$ Table 1 ). However, in their study, E-HPs were not evaluated histopathologically; hence, the proportion of SSA/Ps in E-HPs remained unknown.

In the present study, the overall proportion of SSA/Ps in 792 E-HPs was $2.7 \%$ ( Table 2 ). However, this proportion significantly increased with the size of E-HPs and was significantly higher in the proximal colon than in the distal colorectum. Moreover, no SSA/P was found in the rectum. These findings confirm the knowledge gained in previous studies that the size is a predictor of SSA/P histology and SSA/Ps are predominantly found in the proximal colon [14-17]. If possible, complete resection of all EHPs may be preferable for the prevention of colorectal cancers. However, such an unremitting procedure is not acceptable because of the increased workload for endoscopists and pathologists, the increased medical costs, and the probable adverse events (e.g. bleeding and perforation). Therefore, we propose the resection of all E-HPs apart from distal E-HPs $\leq 5 \mathrm{~mm}$, as an international expert panel recently recommended complete removal of all serrated lesions apart from diminutive sigmoid or rectal lesions [18]. Our proposal is based on the fact that, although a large number of distal E-HPs $\leq 5 \mathrm{~mm}$ were detected, the proportion of SSA/Ps in them was quite low (0.5\%) ( Table 2$)$. If we applied this proposal to the present study, 155 E-HPs would have been resected in $25.4 \%$ of 343 patients, which is acceptable in routine clinical practice. However, in the present study, $3.0 \%$ of resected distal E-HPs $\leq 5 \mathrm{~mm}$ were conventional adenomas. Furthermore, $13.0 \%$ of distal conventional adenomas $\leq 5 \mathrm{~mm}$ were misdiagnosed as HPs, even by high definition magnifying colonoscopy, and these lesions have a risk of becoming interval cancers. Therefore, to avoid leaving neoplastic lesions in situ, we re- commend resecting the distal E-HPs $\leq 5 \mathrm{~mm}$ for which the optical diagnoses are not highly confident.

In the present study, most of the rectosigmoid E-HPs $\leq 5 \mathrm{~mm}$ were not evaluated histopathologically, although SSA/Ps were common, with a prevalence of 5.0\%. Moreover, the prevalence of SSA/Ps was also high (10.2\%) in 59 asymptomatic patients who underwent screening colonoscopy among 343 patients. In previous studies, the prevalence of SSA/Ps has been reported to range from $0.5 \%$ to $13.8 \%$ [15-17,19-23]. This variability is probably due to the differences in study design and population, colonoscopy equipment and observation method, experience of the endoscopists, and the histopathological diagnostic criteria of SSA/Ps. However, it is noteworthy that the prevalence of SSA/Ps was high (13.8\%) in a previous prospective study using chromoendoscopy with indigo carmine dye [15]. Furthermore, Le Rhun et al. reported that pan-colonic chromoendoscopy improves the detection of HPs [12], and indeed, the prevalence of E-HPs was quite high $(85.7 \%)$ in the present study. Therefore, the higher prevalence of SSA/Ps in studies, including our study, using chromoendoscopy than in previous studies using conventional colonoscopy with white light suggests that pan-colonic chromoendoscopy may also improve the detection of SSA/Ps, and SSA/Ps may often be overlooked during conventional colonoscopy. However, pan-colonic chromoendoscopy appears to be unacceptable for routine examinations because of increased costs and procedure time $[10,11]$. In addition, the usefulness of NBI for the detection of colorectal polyps still remains controversial [24]. Therefore, at present, it is necessary to be careful not to overlook E-HPs, particularly in the proximal colon, during conventional colonoscopy.

Moreover, Butterly et al. reported recently that a median normal withdrawal time of 9 min resulted in a statistically significant increase in colorectal adenoma and serrated polyp detection [25]. This is consistent with the mean and median withdrawal times in the present study, which were $9.5 \mathrm{~min}$ and $8.9 \mathrm{~min}$, respectively. Therefore, even though pan-colonic chromoendoscopy cannot be applied to routine examinations, colonoscopy with a median normal withdrawal time of $9 \mathrm{~min}$ may improve the detection rates of E-HPs including SSA/Ps.

In the present study, there was no SSA/P with cytological dysplasia in 343 patients (95\%CI: $0-0.9$ ). Although we cannot exclude the possibility that some SSA/Ps with cytological dysplasia were present in the non-resected distal E-HPs $\leq 5 \mathrm{~mm}$, the prevalence of SSA/Ps with cytological dysplasia has been reported to be quite low $(0.2-0.8 \%$ of patients) in previous studies as well $[16,19-$ 21], which suggests that SSA/Ps with cytological dysplasia may be rare in clinical practice. However, many previous case reports of SSA/Ps with carcinoma, including those showing rapid pro- 
gression, have demonstrated the malignant potential of SSA/Ps [26-29]. Importantly, most of these carcinomas developed in the proximal colon. In addition, it has been reported that interval cancers are more likely to develop in the proximal colon and exhibit microsatellite instability than non-interval cancers [30]. This growing evidence indicates that some SSA/Ps may be overlooked or incompletely resected during colonoscopy, rapidly progress to carcinoma, and lead to interval cancers. Therefore, it is becoming increasingly important to ensure that E-HPs, particularly in the proximal colon, are detected and completely resected during colonoscopy.

This study has a limitation that 3046 rectosigmoid E-HPs in 3683 distal E-HPs $\leq 5 \mathrm{~mm}$ were left in situ. Although complete resection of all E-HPs would be needed to clarify the true proportion of SSA/Ps in E-HPs and the true prevalence of SSA/Ps with or without cytological dysplasia, such a procedure was unfeasible because of the probable adverse events. However, our diagnostic accuracy of differential diagnosis between neoplasia and nonneoplasia for all and rectosigmoid lesions was greater than $90 \%$. Furthermore, the proportion of SSA/Ps in distal E-HPs $\leq 5 \mathrm{~mm}$ was only $0.5 \%$ (95\%CI: $0.1-1.4)($ Table 2$)$. Therefore, we believe that this limitation is unlikely to have affected the proportion of SSA/ Ps in all and distal E-HPs. However, if all E-HPs had been resected in the present study, SSA/Ps would have been more common, with a prevalence of $>5 \%$.

In conclusion, the overall proportion of SSA/Ps in E-HPs was $2.7 \%$. However, this proportion was higher in the proximal colon and increased with the size of E-HPs. SSA/Ps were common in routine colonoscopy, with a prevalence of at least $5.0 \%$. We believe that this knowledge of the prevalence and distribution of varioussized SSA/Ps can help to improve the detection rates and guide the treatment of E-HPs, resulting in the prevention of colorectal cancers arising from SSA/Ps.

\section{Competing interests: None}

\section{References}

$1 \mathrm{Fu}$ KI, Sano Y, Kato S et al. Chromoendoscopy using indigo carmine dye spraying with magnifying observation is the most reliable method for differential diagnosis between non-neoplastic and neoplastic colorectal lesions: a prospective study. Endoscopy 2004; 36: 1089-1093

2 Machida H, Sano Y, Hamamoto Y et al. Narrow-band imaging in the diagnosis of colorectal mucosal lesions: a pilot study. Endoscopy 2004; 36: $1094-1098$

3 Sano Y, Saito Y, Fu KI et al. Efficacy of magnifying chromoendoscopy for the differential diagnosis of colorectal lesions. Dig Endosc 2005; 17 : $105-116$

4 Kimura T, Yamamoto E, Yamano HO et al. A novel pit pattern identifies the precursor of colorectal cancer derived from sessile serrated adenoma. Am J Gastroenterol 2012; 107: 460-469

5 Tadepalli US, Feihel D, Miller KM et al. A morphologic analysis of sessile serrated polyps observed during routine colonoscopy (with video). Gastrointest Endosc 2011; 74: 1360 - 1368

6 Hewett DG, Kaltenbach T, Sano Y et al. Validation of a simple classification system for endoscopic diagnosis of small colorectal polyps using narrow-band imaging. Gastroenterology 2012; 143: 599-607

7 Hayashi N, Tanaka S, Hewett DG et al. Endoscopic prediction of deep submucosal invasive carcinoma: validation of the narrow-band imaging international colorectal endoscopic (NICE) classification. Gastrointest Endosc 2013; 78: 625-632

8 Yao T, Sugai T, Iwashita A et al. Histopathological characteristics and diagnostic criteria of SSA/P, from project research "potential of cancerization of colorectal serrated lesions" of the Japanese Society for Cancer of the Colon and Rectum. Stomach Intestine 2011; 46: 442-448 (in Japanese with English abstract)

9 Fujimori Y, Fujimori T, Imura J et al. An assessment of the diagnostic criteria for sessile serrated adenoma/polyps: SSA/Ps using image pro- cessing software analysis for Ki67 immunohistochemistry. Diagn Pathol 2012; 7: 59

10 Brooker JC, Saunders BP, Shah SG et al. Total colonic dye-spray increases the detection of diminutive adenomas during routine colonoscopy: a randomized controlled trial. Gastrointest Endosc 2002; 56: 333 - 338

11 Pohl J, Schneider A, Vogell $H$ et al. Pancolonic chromoendoscopy with indigo carmine versus standard colonoscopy for detection of neoplastic lesions: a randomized two-centre trial. Gut 2011; 60: 485-490

12 Le RhunM, Coron E, Parlier D et al. High resolution colonoscopy with chromoscopy versus standard colonoscopy for the detection of colonic neoplasia: a randomized study. Clin Gastroenterol Hepatol 2006; 4: $349-354$

13 Yano T, Sano Y, Iwasaki J et al. Distribution and prevalence of colorectal hyperplastic polyps using magnifying pan-mucosal chromoendoscopy and its relationship with synchronous colorectal cancer: prospective study. J Gastroenterol Hepatol 2005; 20: 1572 - 1577

14 Shida Y, Ichikawa K, Fujimori $T$ et al. Differentiation between sessile serrated adenoma/polyp and non-sessile serrated adenoma/polyp in large hyperplastic polyp: A Japanese collaborative study. Mol Clin Oncol 2013; 1: 53-58

15 Spring KJ, Zhao ZZ, Karamatic R et al. High prevalence of sessile serrated adenomas with BRAF mutations: a prospective study of patients undergoing colonoscopy. Gastroenterology 2006; 131: 1400-1407

16 Lash RH, Genta RM, Schuler CM. Sessile serrated adenomas: prevalence of dysplasia and carcinoma in 2139 patients. J Clin Pathol 2010; 63: 681-686

17 Hetzel JT, Huang CS, Coukos JA et al. Variation in the detection of serrated polyps in an average risk colorectal cancer screening cohort. Am J Gastroenterol 2010; 105: 2656-2664

18 Rex DK, Ahnen DJ, Baron JA et al. Serrated lesions of the colorectum: review and recommendations from an expert panel. Am J Gastroenterol 2012; 107: 1315-1329

19 Abdeljawad K, Vemulapalli KC, Kahi CJ et al. Sessile serrated polyp prevalence determined by a colonoscopist with a high lesion detection rate and an experienced pathologist. Gastrointest Endosc; Epub 03.07. 2014

20 Bouwens MW, van Herwaarden YJ, Winkens B et al. Endoscopic characterization of sessile serrated adenoma/polyps with and without dysplasia. Endoscopy 2014; 46: 225 - 235

21 Buda A, De Bona M, Dotti I et al. Prevalence of different subtypes of serrated polyps and risk of synchronous advanced colorectal neoplasia in average-risk population undergoing first-time colonoscopy. Clin Transl Gastroenterol 2012; 3: e6

22 Hazewinkel Y, de Wijkerslooth TR, Stoop EM et al. Prevalence of serrated polyps and association with synchronous advanced neoplasia in screening colonoscopy. Endoscopy 2014; 46: 219-224

$23 \mathrm{Kim} \mathrm{HY,} \mathrm{Kim} \mathrm{SM,} \mathrm{Seo} \mathrm{JH} \mathrm{et} \mathrm{al.} \mathrm{Age-specific} \mathrm{prevalence} \mathrm{of} \mathrm{serrated} \mathrm{le-}$ sions and their subtypes by screening colonoscopy: a retrospective study. BMC Gastroenterol 2014; 14: 82

24 Rex DK, Helbig CC. High yields of small and flat adenomas with highdefinition colonoscopies using either white light or narrow band imaging. Gastroenterology 2007; 133: $42-47$

25 Butterly L, Robinson CM, Anderson JC et al. Serrated and adenomatous polyp detection increases with longer withdrawal time: results from the New Hampshire Colonoscopy Registry. Am J Gastroenterol 2014; 109: $417-426$

26 Ban S, Mitomi H, Horiguchi $H$ et al. Adenocarcinoma arising in small sessile serrated adenoma/polyp (SSA/P) of the colon: clinicopathological study of eight lesions. Pathol Int 2014; 64: 123-132

27 Goldstein NS. Small colonic microsatellite unstable adenocarcinomas and high-grade epithelial dysplasias in sessile serrated adenoma polypectomy specimens: a study of eight cases. Am J Clin Pathol 2006; 125 : $132-145$

28 Sheridan TB, Fenton H, Lewin MR et al. Sessile serrated adenomas with low- and high-grade dysplasia and early carcinomas: an immunohistochemical study of serrated lesions "caught in the act". Am J Clin Pathol 2006; 126: 565-571

29 Oono $Y, F u K$, Nakamura $\mathrm{H}$ et al. Progression of a sessile serrated adenoma to an early invasive cancer within 8 months. Dig Dis Sci 2009; 54: 906-909

30 Arain MA, Sawhey M, Sheikh S et al. CIMP status of interval colon cancers: another piece to puzzle. Am J Gastroenterol 2010; 105: 11891195 